\title{
Supporting questionnaires
}

This supporting information is provided alongside the article: "Case study of Argus in Togo: an SMS and web-based application to support public health surveillance, results from 2016 to 2019".

\section{Healthcare facility user-satisfaction questionnaire}

1. Quel est votre district sanitaire ? $\quad \square$ District $1 \square$ District $2 \square$ District $3 \square$ District $4 \square$ District 5 $\square$ Cinkasse $\square$ Kpendjal $\square$ Tandjaore $\square$ Tone $\square$ Oti

2. Nom complet de la structure sanitaire

3.a) Avez-vous utilisé l'application Argus sur le téléphone pour envoyer des $\square$ Oui $\square$ Non rapports hebdomadaires ou des alertes?

Si De $\mathbf{1}$ (pire) à $\mathbf{5}$ (meilleur), merci de donner un score pour:

OUI 3.b) l'apparence générale d'Argus sur le téléphone $\square 1 \square 2 \square 3 \square 4 \square 5$

3.c) la documentation disponible pour utiliser Argus sur le téléphone $\square 1 \square 2 \square 3 \square 4 \square 5$

3.d) la simplicité d'utilisation globale d'Argus sur le téléphone $\square 1 \square 2 \square 3 \square 4 \square 5$

3.e) la simplicité d'envoi des rapports hebdomadaires avec Argus $\square 1 \square 2 \square 3 \square 4 \square 5$

3.f) I'utilité d'Argus pour l'envoi des rapports hebdomadaires $\square 1 \square 2 \square 3 \square 4 \square 5$

3.g) la simplicité d'envoi des alertes avec Argus $\square 1 \square 2 \square 3 \square 4 \square 5$

3.h) I'utilité d'Argus pour l'envoi des alertes $\square 1 \square 2 \square 3 \square 4 \square 5$

3.i) la simplicité de suivi du statut des précédents rapports et alertes envoyés sur Argus (historique)

Quels problèmes avez-vous rencontrés depuis le début du pilote avec Argus sur le téléphone pour :

3.j) envoyer un rapport hebdomadaire ?

$\square$ Aucun problème

OU description du ou des problème(s) : 
3.k) envoyer une alerte?

$\square$ Aucun problème

OU description du ou des problème(s) :

3.I) suivre le statut des précédents rapports et alertes envoyées (historique) ?

$\square$ Aucun problème

OU description du ou des problème(s) :

3.m) Quelles suggestions pouvez-vous faire pour améliorer Argus sur le téléphone ?

$\square$ Aucune suggestion

OU suggestion(s) d'amélioration(s) :

4.a) Quel est le diplôme de niveau le plus élevé que vous avez obtenu? $\square$ BEPC collège $\square$ BAC lycée $\square$ Assistant d'hygiène d'État

$\square$ Technicien supérieur de santé $\square$ Technicien supérieur de laboratoire

$\square$ Technicien supérieur de génie sanitaire $\square$ Technicien biologiste

$\square$ Infirmier d'État $\square$ Sage-femme d'État $\square$ Médecin

$\square$ Autre, si autre précisez lequel :

\begin{tabular}{|}
\begin{tabular}{|l|l|l|}
\hline 4.a) Avez-vous un téléphone personnel mobile? & $\square$ Oui $\square$ Non \\
\hline Si OUI & 4.b) Depuis combien d'années ? & _- années \\
\hline
\end{tabular}
\end{tabular}

\section{5.a) Avez-vous un téléphone personnel tactile (sans clavier) ? $\quad \square$ Oui $\square$ Non}

Si OUI 5.b) Depuis combien d'années? _ - années 


\section{Intermediate and central-level user-satisfaction questionnaire}

1. Dans quelle structure travaillez-vous?

$\square$ DEPI $\square$ DRS Lomé Commune $\square$ DRS Savanes

$\square$ DPS District $1 \square$ DPS District $2 \square$ DPS District $3 \square$ DPS District $4 \square$ DPS District 5

$\square$ DPS Cinkasse $\square$ DPS Kpendjal $\square$ DPS Tandjaore $\square$ DPS Tone $\square$ DPS Oti

2.a) Avez-vous utilisé le site internet Argus pour valider les rapports

Oui $\square$ Non hebdomadaires ou analyser leurs données?

Si De $\mathbf{1}$ (pire) à $\mathbf{5}$ (meilleur), merci de donner un score pour:

OUI 2.b) l'apparence générale du site internet Argus $\square 1 \square 2 \square 3 \square 4 \square 5$

2.c) la documentation disponible pour utiliser le site internet Argus $\square 1 \square 2 \square 3 \square 4 \square 5$

2.d) la simplicité d'utilisation globale du site internet Argus $\square 1 \square 2 \square 3 \square 4 \square 5$

2.e) la simplicité pour valider les rapports hebdomadaires sur le site $\square 1 \square 2 \square 3 \square 4 \square 5$ internet Argus

$\square$ Ne sait pas

2.f) l'utilité du site internet Argus pour valider les rapports hebdomadaire

$\square 1 \square 2 \square 3 \square 4 \square 5$

2.g) la simplicité pour voir et télécharger les résumés de situation $\square$ Ne sait pas épidémiologique hebdomadaire sur le site internet Argus

2.h) la simplicité pour voir les rapports de promptitude/complétude $\square$ Ne sait pas ainsi que l'évolution du nombre de cas sur le site internet Argus $\square 1 \square 2 \square 3 \square 4 \square 5$

2.i) l'utilité du site internet Argus pour l'analyse des données $\square$ Ne sait pas

$\square 1 \square 2 \square 3 \square 4 \square 5$

Ne sait pas

Quels problèmes avez-vous rencontrés depuis le début du pilote avec le site internet Argus pour :

2.j) valider les rapports hebdomadaires?

$\square$ Aucun problème

OU description du ou des problème(s) : 
3.k) voir et télécharger les résumés de situation épidémiologique hebdomadaire ou voir les rapports de promptitude/complétude ainsi que l'évolution du nombre de cas?

$\square$ Aucun problème

OU description du ou des problème(s) :

3.m) Quelles suggestions pouvez-vous faire pour améliorer le site internet Argus?

$\square$ Aucune suggestion

OU suggestion(s) d'amélioration(s) :

En général, à quelle fréquence utilisez-vous le site internet Argus pour :

2.j) valider les rapports hebdomadaires?

$\square$ une fois par semaine $\square$ une fois toutes les deux semaines $\square$ une fois par mois

$\square$ une fois par trimestre $\square$ une fois par semestre $\square$ une fois par an $\square$ autre, si autre préciser :

Jamais

3.k) pour visualiser et analyser les données?

$\square$ une fois par semaine $\square$ une fois toutes les deux semaines $\square$ une fois par mois

$\square$ une fois par trimestre $\square$ une fois par semestre $\square$ une fois par an $\square$ autre, si autre préciser :

$\square$ Jamais

3.m) pour identifier des évènements de santé publique ?

$\square$ une fois par semaine $\square$ une fois toutes les deux semaines $\square$ une fois par mois

$\square$ une fois par trimestre $\square$ une fois par semestre $\square$ une fois par an $\square$ autre, si autre préciser :

Jamais 


\begin{tabular}{|c|c|c|}
\hline \multicolumn{2}{|c|}{$\begin{array}{l}\text { 4.a) Des investigations ont-elles été déclenchées à partir des données } \\
\text { recueillies par Argus (rapports hebdomadaires et alertes) ? }\end{array}$} & $\square$ Oui $\square$ Non $\square$ Ne sait pas \\
\hline Si OUI & 4.b) Combien depuis le début du pilote & --- \\
\hline
\end{tabular}

4.a) Utilisez-vous le résumé de situation épidémiologique Oui $\square$ Non $\square$ Ne sait pas hebdomadaire pendant les réunions d'équipe?

4.a) Quel est le diplôme de niveau le plus élevé que vous avez obtenu? $\square$ BEPC collège $\square$ BAC lycée $\square$ Assistant d'hygiène d'État

$\square$ Technicien supérieur de santé $\square$ Technicien supérieur de laboratoire

$\square$ Technicien supérieur de génie sanitaire $\square$ Technicien biologiste

$\square$ Infirmier d'État $\square$ Sage-femme d'État $\square$ Médecin

$\square$ Autre, si autre précisez lequel :

\begin{tabular}{|l|l|l|}
\hline 4.a) Avez-vous un ordinateur personnel ? & $\square$ Oui $\square$ Non \\
\hline Si OUI & 4.b) Depuis combien d'années? & __ années \\
\hline
\end{tabular}

5.a) Allez-vous sur internet pour votre usage personnel ?

$\square$ Oui $\square$ Non

\begin{tabular}{l|l} 
Si OUI & $5 . b)$ En général à quelle fréquence?
\end{tabular}

$\square$ Au moins une fois par jour $\square$ Au moins une fois par semaine $\square$ Au moins une fois par mois

Moins de une fois par mois 